EXTENDED REPORT

\title{
Comparing the efficacy and safety of phacoemulsification in white mature and other types of senile cataracts
}

\author{
S S Ermiș, F Öztürk, Ü Ü Inan
}

Br J Ophthalmol 2003;87:1356-1359

See end of article for authors' affiliations

........

Correspondence to: Dr S S Ermis, Dervispasa mah, Pirireis sk, Ceylan Apt $\mathrm{A}$ blok No:3/8 03200 Afyon, Turkey; sametermis@yahoo.com

Accepted for publication 23 February 2003

\begin{abstract}
Aim: To compare the intraoperative difficulty and postoperative outcome in patients who have white mature cataract in one eye and other types of senile cataract in the other eye undergoing clear corneal phacoemulsification and foldable intraocular lens implantation.

Methods: 82 patients who had white mature cataract in one eye, posterior subcapsular, cortical, nuclear, or mixed type cataract in the other eye were enrolled in this prospective study. Postoperative outcomes, intraoperative difficulties related to capsulorhexis, and phacoemulsification were analysed between the two groups of eyes. Postoperative examinations were done at 1 day, 1 week, 1 and 3 months.

Results: Postoperative visual acuity, central corneal thickness, intraocular pressure, and rate of posterior capsule rupture were not significantly different between the two group of eyes ( $p>0.05$ ). Mean effective phaco time, frequency of postoperative corneal oedema and posterior capsular plaque were found to be significantly higher in the mature cataract group $(p<0.05)$.

Conclusions: A one stage, $5 \mathrm{~mm}$ continuous capsulorhexis was achieved using trypan blue and generous amounts of retentive viscoelastic agent in eyes with white mature cataract. Intraoperative difficulties and postoperative outcome of clear corneal incision phacoemulsification surgery and foldable intraocular lens implantation were similar in white mature and other types of senile cataract. Topical anaesthesia in phacoemulsification of eyes with white mature cataract is safe and well tolerated.
\end{abstract}

\section{PATIENTS AND METHODS}

A total of 82 patients (43 women and 39 men) who had bilateral senile cataracts with a mean age of 67.7 (SD 9.5) years (range 49-85) were enrolled in this prospective study. Patients had white mature cataract in one eye (mature cataract group), posterior subcapsular, cortical, nuclear or mixed type cataract in the other eye (control group). All eyes in mature cataract group lacked red fundus reflex. Cataracts appearing white on slit lamp examination were defined as white mature cataracts. Patients with diabetes mellitus, glaucoma, pseudoexfoliation, complicated, brunescent, black, or hypermature (Morgagnian) cataract, poor pupil dilatation $(<5.0 \mathrm{~mm})$, history of ocular surgery, laser treatment, or trauma were excluded. All surgery was performed between June 2001 and June 2002.

Preoperative ocular examinations included Snellen visual acuity, detailed biomicroscopic examination including anterior chamber cell count measurement (graded from 0 to 3 and was evaluated by one investigator), Goldmann applanation tonometry, axial length (AL) and anterior chamber depth (ACD) measurements with A-scan ultrasonography (Ophthasonic, Mentor, Teknar), central corneal thickness (CCT) measurements with ultrasonic pachymeter (BVI, Quantel Medical). Keratometry was performed using an autokeratorefractometer (Topcon KR-7000P). Postoperative examinations were done at 1 day, 1 week, 1 and 3 months. Written informed consent was obtained from each patient.

Nuclear hardness was subjectively evaluated by the surgeon intraoperatively during phacoemulsification and was graded as 0 : soft, 1 : semi soft, 2: medium to hard, 3: hard, 4: very hard. Effective phaco time displayed by the phacoemulsification unit for each surgical procedure was recorded.

Cyclopentolate $1 \%$ and phenylephrine $2.5 \%$ eye drops were used for mydriasis four times 1 hour before the surgery. Endocapsular phacoemulsification was performed in all eyes by using Diplomax (Allergan) phacoemulsification unit by one of the three surgeons in an identical manner. Topical or retrobulbar anaesthesia was used. Pieces of cell sponge which are impregnated with oxybuprocaine (proparacaine) $\mathrm{HCl}$ $0.5 \%$ (Alcaine, Alcon) were placed deep into the superior and inferior fornix for 15 minutes before surgery for topical anaesthesia. Retrobulbar injection was performed using a 23 gauge needle; $3 \mathrm{ml}$ of $2 \%$ lidocaine was given intraconally. A three step clear corneal tunnel incision was made with a $3.2 \mathrm{~mm}$ disposable metal blade and a side port incision was 
made with an MVR blade. After injection of sodium chondroitin sulfate-sodium hyaluronate (Viscoat, Alcon) into the anterior chamber of eyes with white cataracts $0.1 \mathrm{ml}$ of trypan blue $0.1 \%$ (trypan blue $0.4 \%$ in $0.81 \%$ sodium chloride solution, cell culture tested, Sigma; diluted with BSS) was injected onto the anterior capsule slowly. Simultaneously the dye was spread mechanically with the anterior chamber cannula to obtain a homogeneous and diffuse staining of the anterior capsule. Additional sodium chondroitin sulfatesodium hyaluronate was given in order to deepen the anterior chamber and obtain anterior chamber clarity. The dye was used for all white mature cataracts which lacked red fundus reflex. A central initial puncture of the anterior capsule was made with a 26 gauge needle, and the needle was moved to the left approximately $2.0 \mathrm{~mm}$ in order to create a capsular flap. Uttrata forceps were used to grasp the capsule and perform a capsulorhexis of $5.0 \mathrm{~mm}$ diameter. Gentle hydrodissection was made and the nucleus was rotated with an anterior chamber cannula. If the capsulorhexis tear was directed towards the periphery more viscoelastic was given onto the anterior capsule at that location or Vannas scissors was used to prevent uncontrolled extension. Endocapsular phacoemulsification was performed using a quadrant divide and conquer technique in eyes with white cataracts, chip and flip technique in contralateral eyes. A 15 degree bevel tip was used for all cases. Cortical remnants were removed by an automated irrigating/aspiration hand piece of the phaco unit, anterior and posterior capsular polishing was performed. Corneal incision was enlarged to $4.0 \mathrm{~mm}$ with a metal blade, a foldable hydrophilic acrylic intraocular lens with a $12.0 \mathrm{~mm}$ overall size and a $5.75 \mathrm{~mm}$ optic (Soft Tec I, Lenstec) was implanted intracapsularly and the viscoelastic was removed. The anterior chamber was inflated using a balanced salt solution. The corneal incision was checked for water tightness and left unsutured. Postoperatively all patients used ofloxacin $0.3 \%$ and prednisolone acetate $1 \%$ eye drops four times a day for 1 month.

Student's $t$, Fischer exact test, and correlation analyses were used for statistical analyses. A p value $<0.05$ was considered significant.

\section{RESULTS}

While retrobulbar anaesthesia was performed initially, topical anaesthesia was used for the last 25 cases in the mature cataract group and last 33 cases in the control group. None of the patients complained of intolerable pain under topical or retrobulbar anaesthesia. One stage continuous capsulorhexis was successfully completed in all cases under topical anaesthesia. A posterior capsular tear occurred in one eye in the mature cataract group in which topical anaesthesia was used. In the mature cataract group mean effective phaco time was 44.3 (SD 30.9) seconds in cases in which retrobulbar anaesthesia was performed and 37.1 (15.9) seconds with topical anaesthesia, the difference was not significant $(p=0.28)$. In the control group the mean effective phaco time was 20.3 (11.5) seconds with retrobulbar anaesthesia, 21.8 (10.4) seconds with topical anaesthesia, the difference was not significant $(\mathrm{p}=0.55)$.
In all cases in the mature cataract group the anterior capsule stained adequately for visibility during capsulorhexis. Slight staining of the corneal and side port incision site cleared after irrigation/aspiration of cortical material. No residual coloration of the posterior capsule was noted after removal of lens material. In the mature cataract group capsulorhexis was performed successfully using only Uttrata forceps and without the need of additional viscoelastic in 69 eyes. In 13 cases which developed uncontrolled extension of capsulorhexis more viscoelastic was given onto the capsule or Vannas scissors was used to prevent the tear edge going to periphery. Despite to that, peripheral extension to the equator occurred in two eyes; we returned to the starting point of the capsulorhexis, proceeded with a second tear in the opposite direction and completed the capsulorhexis. Capsulorhexis was successfully completed using only Uttrata forceps and without additional viscoelastic in all eyes in the control group.

Posterior capsule tear occurred in three eyes in the mature cataract group and two eyes in the control group, all at the nuclear fragment consumption stage, the difference was not significant (Fisher's exact test, $p=1.0$ ). One eye which developed posterior capsular tear in the mature cataract group had previous peripheral extension of capsulorhexis. Intraocular lens was inserted intracapsularly in all patients with posterior capsule tear except the case which had previous capsulorhexis discontinuity. A sulcus fixated polymethyl methacrylate intraocular lens $(6.0 \times 13.0 \mathrm{~mm})$ was implanted in this case. Postoperative visual acuity at 3 months was 0.6 or better in patients with posterior capsular tear.

Posterior capsular plaque was noted in 10 eyes $(12.2 \%)$ in the mature cataract group and in two eyes $(2.4 \%)$ in the control group; the difference was statistically significant (Fisher's exact test, $\mathrm{p}=0.032$ ).

Twenty one eyes had posterior subcapsular, 29 eyes had cortical, 18 eyes had nuclear, and 14 eyes had mixed type cataract in control group. Red fundus reflex was adequate to perform capsulorhexis in all eyes in this group.

In the mature cataract group preoperative visual acuity was at the level of hand movements in all cases, mean postoperative visual acuity was $0.84(0.17)$ at 3 months. In the control group mean preoperative visual acuity was 0.24 (0.12), improved to $0.88(0.18) 3$ months after the surgery. The difference between postoperative visual acuity levels between two groups was not significant $(p=0.15)$.

Table 1 shows the AL, ACD and keratometry of the eyes. While preoperative ACD was significantly smaller in the mature cataract group $(\mathrm{p}<0.01), \mathrm{AL}$, keratometry, and postoperative ACD at 3 months did not differ significantly between the groups.

Mean effective phaco time was 40.4 (23.6) seconds in the mature cataract group, 21.1 (10.7) seconds in control group, the difference was statistically significant $(p<0.001)$. Mean nuclear hardness score was $2.4(0.6)$ in the mature cataract group, $1.7(0.7)$ in the control group; the difference was significant $(p<0.001)$. There was a statistically significant correlation between effective phaco time and nucleus hardness

Table 1 Axial length (AL), anterior chamber depth (ACD), and mean keratometry reading $(\mathrm{K})$ of the cases

\begin{tabular}{llll}
\hline & Mature cataract group & Control group & p Value \\
\hline AL $(\mathrm{mm})$ & $23.48(1.86)$ & $23.61(1.62)$ & 0.63 \\
Preop ACD $(\mathrm{mm})$ & $2.77(0.49)$ & $3.32(0.38)$ & $<0.01$ \\
Postop ACD $(\mathrm{mm})$ & $3.47(0.47)$ & $3.44(0.35)$ & 0.64 \\
Preop K (dioptres) & $42.88(1.48)$ & $42.84(1.65)$ & 0.87 \\
\hline
\end{tabular}




\begin{tabular}{llll} 
Table 2 & Preoperative and postoperative intraocular pressure $(\mathrm{IOP})$ & of cases $(\mathrm{mm} \mathrm{Hg})$ \\
\hline & Mature cataract group & Control group & p Value \\
\hline Preoperative & $13.23(3.99)$ & $12.73(3.31)$ & 0.38 \\
Postoperative & $14.30(3.99)$ & $13.70(3.73)$ & 0.32 \\
1 day & $12.59(4.06)$ & $11.86(3.55)$ & 0.22 \\
1 week & $12.45(4.25)$ & $11.54(3.65)$ & 0.14 \\
1 month & $12.84(3.56)$ & $12.20(3.69)$ & 0.26 \\
3 months & &
\end{tabular}

score in both groups $(r=0.76, \mathrm{p}=0.01$ in the mature cataract group; $r=0.83, \mathrm{p}=0.01$ in the control group).

Mean preoperative and postoperative intraocular pressure values in both groups are seen in Table 2. The difference between the groups was not significant at preoperative and all postoperative visits.

Anterior chamber cell score was 1.64 (0.95) in the mature cataract group, $1.73(0.99)$ in the control group at 1 day postoperative visit. There was no significant difference between the two groups $(\mathrm{p}=0.55)$.

At 1 day postoperative visit corneal oedema was present in 17 eyes $(20.7 \%)$ in the mature cataract group and three eyes $(3.7 \%)$ in the control group, the difference was significant (Fisher's exact test, $\mathrm{p}=0.01$ ). Anti-oedema treatment with $5 \%$ sodium chloride solution was used and oedema resolved 1 week after surgery in all eyes.

Table 3 shows the CCT values. There was no statistical difference between the groups throughout the study. No correlation was found between effective phaco time and increase in CCT at the first postoperative day in both groups $(r=0.027, \mathrm{p}=0.906$ in mature cataract group; $r=032$, $\mathrm{p}=0.143$ in control group). CCT was 621 (35) in eyes with corneal oedema and 602 (31) in eyes without corneal oedema at the first postoperative day in mature cataract group, the difference was statistically significant $(p=0.031)$. When cases with and without corneal oedema were evaluated together in mature cataract group and compared with control group the difference was insignificant $(\mathrm{p}=0.75)$.

Slight thermal injury of the corneal incision site was noted in two eyes in the mature cataract group. No suture was used and there was no leakage of fluid at the end of the surgery and postoperative controls. The intraoperative and postoperative complications in two groups of eyes are shown in Table 4.

\section{DISCUSSION}

Johnston et al compared topical and peribulbar anaesthesia for phacoemulsification and reported no significant difficulty or complication under topical anaesthesia. ${ }^{7}$ In the white mature cataract group we observed no difficulty in performing capsulorhexis under topical anaesthesia and all steps of the surgery were comfortable for the surgeon as they are with retrobulbar anaesthesia. Topical anaesthesia requires cooperative patients; sudden eye movements may be dangerous when performing capsulorhexis and using pointed instruments in the eye. The surgeon must be ready to immobilise the globe with a second instrument.

General recommendations for visualisation of the anterior capsule in eyes with white mature cataracts include dimming the operation room lights, increasing the magnification of microscope, using oblique illumination, using an endoilluminator, ${ }^{8}$ capsule dyes, and performing a two stage capsulorhexis. ${ }^{1249}$ In our cases, staining the capsule with trypan blue under viscoelastic material enhanced the visualisation of the anterior capsule during capsulorhexis. Although originally Melles et al ${ }^{10}$ stained the capsule under an air bubble it was reported that using the dye under a dispersive viscoelastic material was easier and safe. ${ }^{11}$ The air bubble technique was reported to be time consuming and it was difficult to reform the anterior chamber by a single air bubble. ${ }^{11}$ Neither viscoelastic exchange nor irrigation was required with the concentration of trypan blue we used. We injected only a small amount of additional viscoelastic material into the anterior chamber to replace the portion of viscoelastic material that escaped during capsule staining manipulation and to obtain anterior chamber clarity. Viscoelastic exchange and irrigation steps prolong surgery and require extra anterior chamber manipulations. Additionally, the enhanced visualisation of the anterior capsule helped the surgeon to identify the capsule edge from the underlying white cortical material and avoid operating trauma to the capsulorhexis edge during phacoemulsification.

The difficulties in performing capsulorhexis in eyes with mature cataracts were absence of red fundus reflex, leakage of fluid immediately as the capsule is punctured, fragility of anterior capsule. Additionally intracapsular pressure remains high even after the initial puncture and the tear tends to extend to the periphery and it becomes difficult to control the capsulorhexis. ${ }^{3}$ We achieved a one stage, $5 \mathrm{~mm}$ continuous capsulorhexis in most of the cases.

Although hydrodissection was not recommended in white mature cataracts, ${ }^{1}$ we observed that gentle hydrodissection broke the corticocapsular adhesions that could resist free nucleus rotation. Singh et $a l^{12}$ reported corticocapsular adhesions resulted in difficult nucleus rotation in brunescent and black cataracts. Nucleus rotation is crucial for phacoemulsification.

Posterior capsule presents increased challenges in the surgery of an intumescent lens. Apart from more prolonged

Table 3 Preoperative and postoperative central corneal thickness (CCT) of cases

\begin{tabular}{|c|c|c|c|}
\hline & Mature cataract group & Control group & p Value \\
\hline $\begin{array}{l}\text { Preoperative } \\
\text { Postoperative }\end{array}$ & $537(33)$ & $539(31)$ & 0.69 \\
\hline 1 day & $606(37)$ & $604(44)$ & 0.75 \\
\hline 1 week & 575 (43) & $570(34)$ & 0.41 \\
\hline 1 month & $555(40)$ & $550(38)$ & 0.41 \\
\hline 3 months & 544 (37) & 538 (35) & 0.29 \\
\hline
\end{tabular}


Table 4 Intraoperative and postoperative complications

\begin{tabular}{lll}
\hline Complications & $\begin{array}{l}\text { Mature cataract } \\
\text { group }\end{array}$ & $\begin{array}{l}\text { Control } \\
\text { group }\end{array}$ \\
\hline Capsulorhexis discontinuity & 2 & 0 \\
Posterior capsular rupture & 3 & 2 \\
Wound site thermal injury & 2 & 0 \\
Corneal oedema & 17 & 3 \\
\hline
\end{tabular}

phacoemulsification time and manipulation of a large and hard nucleus, the posterior capsule is often thinned and stretched by the expanded intumescent lens. As a result, posterior capsule is not only weak, but also flaccid with wrinkles and a laxity that makes it prone to be ruptured during phacoemulsification particularly during nuclear fragment consumption stage. The problem is worsened by the absence of any epinucleus that protects the posterior capsule. A useful step is to inject a dispersive, non-cohesive viscoelastic behind the nucleus during phacoemulsification which will provide an artificial epinucleus to keep the posterior capsule back from the operating plane and also stabilise the nucleus against tumbling. In our study, with posterior capsule rupture, there was no statistically significant difference between the groups. We observed posterior capsule rupture in one eye with previous capsulorhexis discontinuity. During the phaco, the surgeon can displace the nucleus away from the tear in the continuity of the capsulorhexis and this area should be reserved for the final manoeuvres during cortical aspiration.

In brunescent and black cataracts the lens fibres were found to be very cohesive thus making division difficult. ${ }^{12}$ White cataracts in our study were usually brittle and not very hard; they were safely divided and emulsified; but in a few cases leathery fibres kept most of the nucleus joined. There was also a tendency for hardening of the lens as the duration of visual symptoms increased.

In our cases we did not encounter complications of capsular fibrosis and geometrical decentration. In another study capsular fibrosis was reported to occur in $12 \%$ of eyes with white mature cataracts, all of which had a capsulorhexis diameter of less than $5 \mathrm{~mm} .^{2}$ Small capsulorhexis was reported to lead to capsule contraction. ${ }^{13}{ }^{14}$

Both group of eyes in our study had an increase in CCT on the first postoperative day (Table 3). CCT returned to preoperative level after 3 months. Studies reported similar results after phacoemulsification of standard, white mature, brunescent and black cataracts. ${ }^{2}{ }^{1516}$ Although corneal oedema resolved in all cases 1 week after the surgery, it may be important to inform patients about the possibility of a transient reduction in vision for a few days.

In our study, intraoperative examination of the posterior capsule showed the presence of plaques in $12.2 \%$ of the eyes. Previous studies reported this ratio as $27.3 \%$ and $33 \% .^{25}$ This difference may be related to the time that the patients waited for the surgery.

In developed countries it may be an obligation for the surgeon to answer the question: "Why did the patient wait so long before presenting?" and investigate the potential value of surgery; but in developing countries white mature cataracts may constitute a significant proportion of the patients with cataract. There may be little need for extensive examinations.

In conclusion, we achieved a one stage, $5 \mathrm{~mm}$ continuous capsulorhexis using trypan blue and generous amount of retentive viscoelastic agent. Intraoperative difficulties and postoperative outcome of clear corneal incision phacoemulsification surgery and foldable intraocular lens implantation were similar in white mature and other types of senile cataract. Topical anaesthesia in phacoemulsification of eyes with white mature cataract is safe and well tolerated.

\section{Authors' affiliations}

S S Ermis, F Öztürk, Ü Ü Inan, Department of Ophthalmology, School of Medicine, University of Afyon Kocatepe, Afyon, Turkey

None of the authors has any proprietary interest in any product mentioned.

\section{REFERENCES}

1 Chakrabarti A, Singh R. Phacoemulsification in eyes with white cataract. J Cataract Refract Surg 2000;26:1041-7.

2 Vasavada A, Singh R, Desai J. Phacoemulsification of white mature cataracts. J Cataract Refract Surg 1998;24:270-7.

3 Vasavada A, Singh R. Surgical techniques for difficult cataracts. Curr Opin Ophthalmol 1999; 10:46-52.

4 Gimbel HV, Willerscheidt $A B$. What to do with limited view: the intumescent cataract. J Cataract Refract Surg 1993; 19:657-61.

5 Vasavada A, Chauhan H, Shah G. Incidence of posterior capsular plaque in cataract surgery. J Cataract Refract Surg 1997;23:798-804.

6 Leite E. Rock hard cataract: the major challenge for phacoemulsification. In: Buratto L, Osher R, Masket S, eds. Cataract surgery in complicated cases. Thorofare, NJ: Slack, 2000:37-48

7 Johnston RL, Whitefield LA, Giralt J, et al. Topical versus peribulbar anesthesia without sedation, for clear cornea phacoemulsification. J Cataract Refract Surg 1998;24:407-10.

8 Mansour AM. Anterior capsulorhexis in hypermature cataract (letter). $J$ Cataract Refract Surg 1993;19:116-17.

9 Gimbel HV. Two-stage capsulorhexis for endocapsular phacoemulsification. J Cataract Refract Surg 1990;16:526-9.

10 Melles GR, de Waard PW, Pameyer JH, et al. Trypan blue capsule staining to visualize the capsulorhexis in cataract surgery. J Cataract Refract Surg 1999;25:7-9.

11 Yetik H, Devranoglu K, Ozkan S. Determining the lowest trypan blue concentration that satisfactorily stains the anterior capsule. J Cataract Refract Surg 2002;28:988-91.

12 Singh R, Vasavada A, Janaswamy G. Phacoemulsification of brunescent and black cataracts. J Cataract Refract Surg 2001;27:1762-9.

13 Davison JA. Capsule contraction syndrome. J Cataract Refract Surg 1993;19:582-9.

14 Masket S. Postoperative complications of capsulorhexis. J Cataract Refract Surg 1993;19:721-4.

15 Ventura AC, Walti R, Böhnke M. Corneal thickness and endothelial density before and after cataract surgery. Br J Ophthalmol 2001;85:18-20.

16 Ravalico G, Tognetto D, Palomba MA. Corneal endothelial function after extracapsular cataract extraction and phacoemulsification. J Cataract Refract Surg 1997;23:1000-5. 